\title{
A Review on Prostate Cancer Detection using Deep Learning Techniques
}

\author{
1Narmatha C, ${ }^{2}$ Surendra Prasad M \\ ${ }^{1}$ Department of Computer Science, Faculty of Computers and Information Technology, University of Tabuk, Saudi Arabia \\ Annapoorana Medical College and Hospitals. Salem, Tamil Nadu, India \\ **Corresponding Author: surendar1745@gmail.com
}

Received: 10.06 .2020 Revised: 10.07.2020, Accepted: 19.08 .2020 , Published: 31.09 .2020 DOI:

10.53409/mnaa.jcsit20201204
Abstract: The second most diagnosed disease of men throughout the world is Prostate cancer (PCa). $28 \%$ of cancers in men result in the prostate, making PCa and its identification an essential focus in cancer research. Hence, developing effective diagnostic methods for PCa is very significant and has critical medical effect. These methods could improve the advantages of treatment and enhance the patients' survival chance. Imaging plays a significant role in the identification of PCa. Prostate segmentation and classification is a difficult process, and the difficulties fundamentally vary with one imaging methodology then onto the next. For segmentation and classification, deep learning algorithms, specifically convolutional networks, have quickly become an optional technique for medical image analysis. In this survey, various types of imaging modalities utilized for diagnosing $\mathrm{PCa}$ is reviewed and researches made on the detection of PCa is analyzed. Most of the researches are done in machine learning based and deep learning based techniques. Based on the results obtained from the analysis of these researches, deep learning based techniques plays a significant and promising part in detecting PCa. Most of the techniques are based on computer aided detection (CAD) systems, which follows preprocessing, segmentation, feature extraction, and classification processes, which yield efficient results in detecting PCa. As a conclusion from the analysis of some recent works, deep learning based techniques are adequate for the detection of PCa.

Keywords: Prostate Cancer, CAD system, MRI, Ultrasound, Segmentation, Classification

\section{INTRODUCTION}

PCa is the second common diagnosed disease and one of the critical diseases to cause mortality among men around the world. The growth of overall $\mathrm{PCa}$ is expected to reach around 2.3 million new cases and 740000 deaths by 2040 normally because of the aging and growth of the population. In view of the GLOBOCAN database 2018, the incidence and mortality rate is represented in figure 1 and 2 [1].

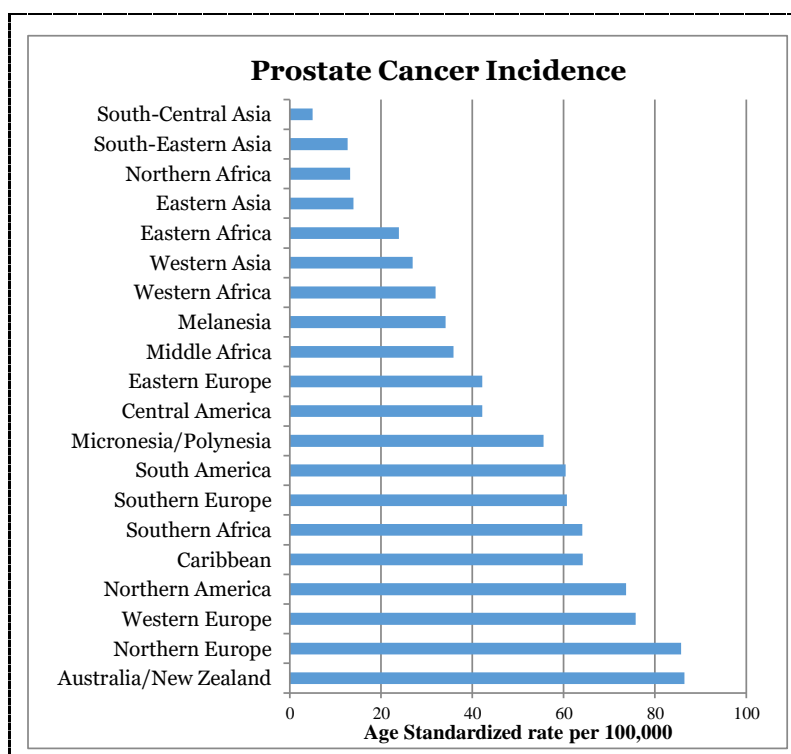

Figure.1. Prostate Cancer Incidence Chart of 2018

In 2018, the number of new cases recorded all over the world was $1,276,106$, which covered $7.1 \%$ of cancer patients excluding other cancers 
globally. And 358,989 deaths were occurred in 2018 globally due to $\mathrm{PCa}$, which covers $3.8 \%$ of mortality. It is one of frequently diagnosing cancer around men in more than one-half (105 of 185) of the world nations.

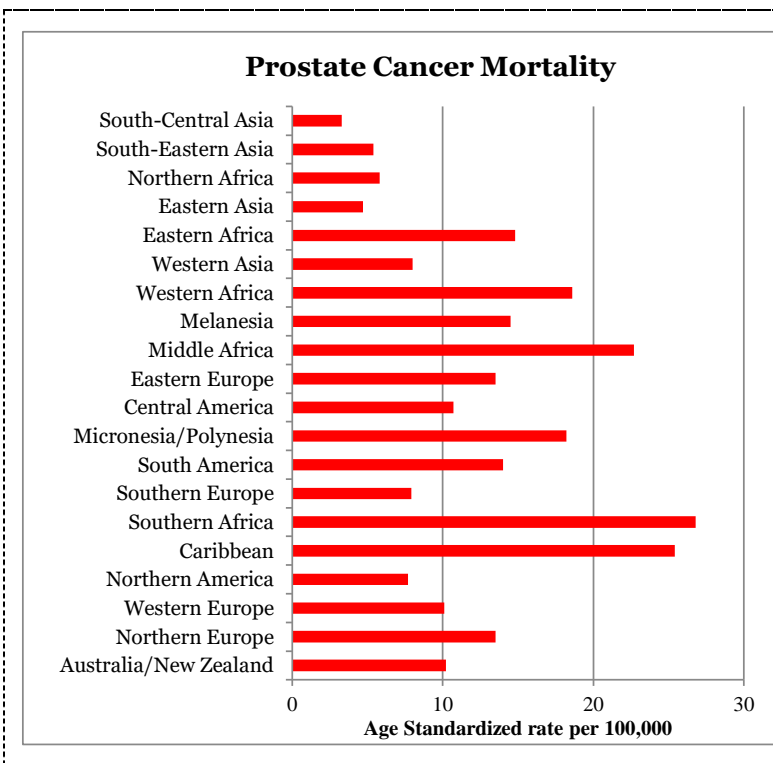

Figure.2. Prostate Cancer Mortality Chart of 2018

The higher incidence rate and death rates worldwide are shown in figure.2. $\mathrm{PCa}$ has conventionally been diagnosed by digital rectal exam (DRE) and prostate-specific antigen (PSA) blood test, trailed by transrectal ultrasound (TRUS) or multi-parametric MRI (mpMRI) guided biopsy. As PCa is a multiform disease, extending from small, slow, low-grade tumors, to large, intense, dangerous tumors, the essential objective for urologists during baseline assessment of prostate disease is, subsequent to determining the presence of the tumor, to assess local and distant cancer expansion, and its grade by staging [2]. However, Transrectal Ultrasound (TRUS) directed biopsy was as yet the principle methodology for diagnosing PCa [3], it was not suggested because of its intrusiveness [4].

Rapid technological advances in the course of the most recent years have empowered the standard utilization of imaging prostate for the clinical direction of PCa. Imaging models like multiparametric-MRI (mpMRI), multiparametric ultrasound (mpUS), and Positron Emission Tomography (PET) nuclear imaging were currently being utilized for every aspect of $\mathrm{PCa}$ diagnoses and localizations, staging, focal and whole-gland therapy, recurrence monitoring, and active surveillance. MRI has demonstrated as a major compelling imaging method for $\mathrm{PCa}$, enhancing localizations and direction of biopsies, particularly for anterior cancers [5]. T1 \& T2 stage infection that was bound to the prostate was effectively perceptible on MRI, while additional prostatic diseases ( $\mathrm{T}_{3}$ and $\mathrm{T}_{4}$ ) were difficult for visualization.

Table.1. Advantages and Disadvantages of Imaging Modalities

\begin{tabular}{|c|c|c|c|c|}
\hline $\begin{array}{l}\text { Imaging } \\
\text { Models }\end{array}$ & Clinical Usage & Advantages & Drawbacks & Prospective \\
\hline $\begin{array}{l}\text { Ultrasound- } \\
\text { based }\end{array}$ & $\begin{array}{l}\text { Early diagnosis and } \\
\text { detection }\end{array}$ & $\begin{array}{l}\text { Widely available, Office- } \\
\text { based, real-time imaging, } \\
\text { inexpensive }\end{array}$ & $\begin{array}{l}\text { Limited tissue contrast among } \\
\text { cancerous and benign tissue }\end{array}$ & $\begin{array}{l}\text { mpUS-based method } \\
\text { (RTE, CEUS) may } \\
\text { enhance contrast }\end{array}$ \\
\hline mpMRI-based & $\begin{array}{l}\text { Early diagnosis and } \\
\text { recurrence, active } \\
\text { surveillance, staging, } \\
\text { metastatic } \\
\text { involvement }\end{array}$ & $\begin{array}{l}\text { Best tissue contrast for } \\
\text { detection of medically } \\
\text { significant Prostate } \\
\text { Cancer }\end{array}$ & $\begin{array}{l}\text { High-cost due to in-bore time, } \\
\text { lack of real-time imaging, needs } \\
\text { advanced training }\end{array}$ & $\begin{array}{l}\text { Alternative in-bore } \\
\text { options with real-time } \\
\text { imaging being advanced }\end{array}$ \\
\hline $\begin{array}{l}\text { mpMRI- } \\
\text { ultrasound } \\
\text { fusion-based }\end{array}$ & $\begin{array}{l}\text { Early diagnosis and } \\
\text { detection, active } \\
\text { surveillance }\end{array}$ & $\begin{array}{l}\text { Combines multimodality } \\
\text { data, Office-based }\end{array}$ & $\begin{array}{l}\text { Most costly, needs either } \\
\text { fusion-device specific training } \\
\text { or ample experience to execute } \\
\text { cognitive fusion, registration } \\
\text { errors during MRI-ultrasound } \\
\text { fusion }\end{array}$ & $\begin{array}{l}\text { Gaining popularity } \\
\text { worldwide, but additional } \\
\text { enhancements to mini- } \\
\text { mize registration errors } \\
\text { required }\end{array}$ \\
\hline PET-based & $\begin{array}{l}\text { Staging, recurrence, } \\
\text { metastatic spread }\end{array}$ & $\begin{array}{l}\text { Offers ancillary details } \\
\text { for tumor staging, } \\
\text { characterization and } \\
\text { metastatic involvement }\end{array}$ & $\begin{array}{l}\text { High-cost, technological (i.e. } \\
\text { attenuate +-ion correction) } \\
\text { and/or medical challenges (i.e. } \\
\text { radiation effects) }\end{array}$ & $\begin{array}{l}\text { Development of specific } \\
\text { radionuclides is an } \\
\text { ongoing endeavor }\end{array}$ \\
\hline
\end{tabular}

As a result of disease's variability, analysts and clinicians in recent years have begun utilizing multiparametric MRI (mpMRI), consolidating anatomic and functional imaging by many other successions to give a progressively comprehensive image. The mpMRI diagnosis commonly comprise diffusion weighted imaging (DWI) for cellularity, T2-w for anatomy, and for vascularity, dynamic contrast enhanced MRI (DCE-MRI) [6]. Various clinical analyses have evaluated the efficiency of mpMRI for $\mathrm{PCa}$, and have decided it was the finest non-invasive alternation for detecting cancer. The mpMRI has can alter the ideal models on PCa detection and classification of risk [7]. The mpMRI of the prostate was basically some operative imaging 
types utilized to complement normal anatomical $\mathrm{T} 1$ and T2-weighted images. The operative arrangements of options are DWI and DCE, Imaging plays an inexorably significant part in the initial recognition and the direction of $\mathrm{PCa}$. The key imaging models, mpMRI, mpUS, PET, and MRI-US fusion are utilized in the localization and diagnosing PCa. Significance was based on conditions of tumors biologically that maintain the utilization of particular imaging methods [9]. PCa diagnosis was generally depended on various methods as digital rectal examination, PSA, TRUS, MRI, and transrectal biopsy. Specifically, the DWI-MRI method enables for acquiring images with contrast contingent upon the tissue's microscopic water molecules mobility, testing the microscopic structures. Additionally from DWI, image was conceivable for evaluating the Apparent comprising the apparent diffusion co-efficient (ADC) maps calculation [8].

Diffusion Coefficient (ADC) of water utilizing diverse diffusion model, as "Monoexponential", "Bi-exponential", "Kurtosis", "Gamma conveyance" and "Stretched exponential", each one of them dependent on various speculations on the tissue's microenvironment water mobility [10] [12]. Multi-parametric MRI could present detailed representation of prostate lesions and tissues. The cancer could be recognized earlier any essential invasive methodologies like needle biopsy, at the risk of harm or periprostatic nerves irritation, bladder neck and prostate. In any case, the prostate tissue cancer on MRI could likewise be hard to detect, with frequently uncertain results between the clinicians [13-14].

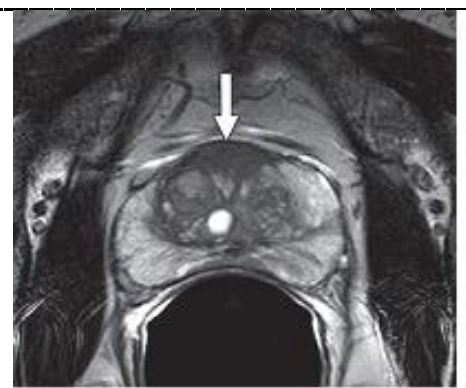

a
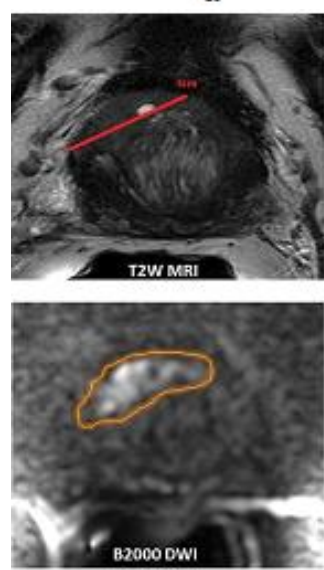

d

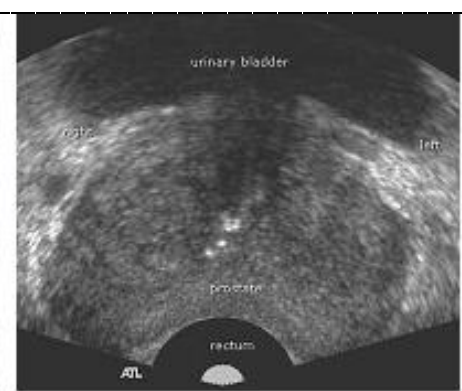

b
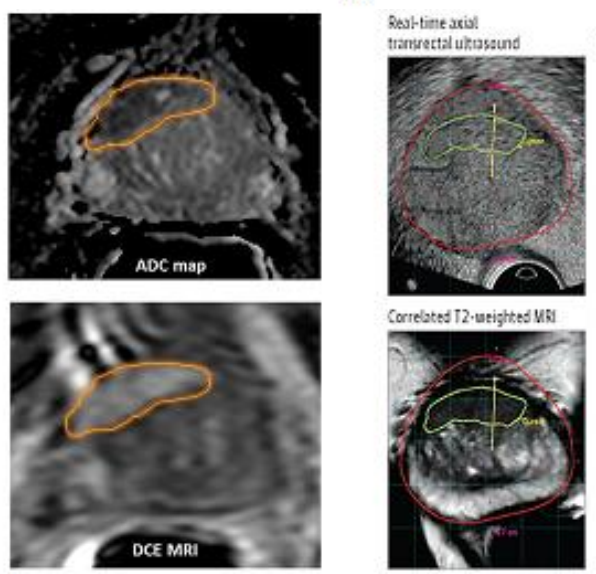

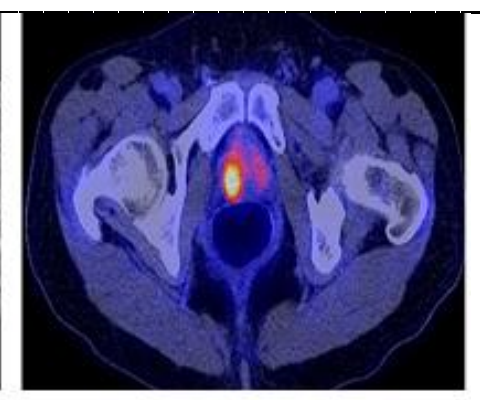

c

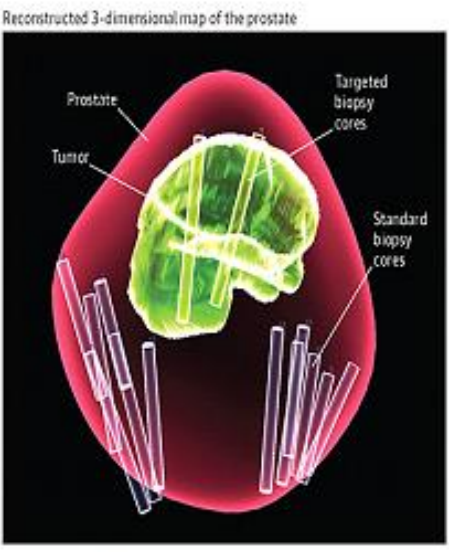

e

Figure.3. Diagnosis of Prostate Cancer using, a) MRI, b) Ultrasound, c) PET, d) mp-MRI, and e) MRI-US fusion [10]

\section{RELATED WORK BASED ON SEGMENTATION AND CLASSIFICATION}

\subsection{Segmentation based Analysis}

Segmenting PCa was a difficult assignment, and the difficulties fundamentally vary starting with one imaging methodology then onto the next. Micro-calcifications, speckle, low contrast, and imaging artifacts such as shadow presents critical difficulties to segment exact prostate in TRUS. Be that as it may, in MRI, superior soft tissues contrast emphasizes huge variations in size, texture, and shape data within the prostate. Interestingly, poor soft tissue differentiates among the prostate and encompassing tissue in CT image makes difficulty in segmenting precise prostate segmentation [15] [16]. 
Precise and solid prostate gland segmentation utilizing MRI has the significance in diagnosing and medication of prostate cancer. Although several automatic segmentation techniques depended on deep learning and machine learning has been proposed, the performance of segmentation has opportunity to get better because of the huge variation in image appearance, interference, and anisotropic spatial resolutions. A $3 \mathrm{D}$ adversarial pyramid anisotropic convolutional deep neural network for segmenting MRI prostate was used in [17]. The FCNN-based network structures for segmenting MRI PCa image was introduced in [18] and analyzed different structures of alternate associations along with the deep network size and recommend 8 diverse FCNNbased deep 2D network structure for automated MRI segmentations of prostate. In [19], an ACM (Active Contour Model) was trained and utilized for segmenting the PCa. Then, after injection, features were extricated from the dynamic MRIs at various times and changed them into RIC curve. Then, discriminative features were chosen by SFFS (Sequential Forward Floating Selection) and FDR (Fisher's Discrimination Ration).

By using the Salp Swarm Optimization Algorithm-based Rider Neural Network (SSARideNN) and Color Space (CS) transformation the segmentation and classification was done in [20]. Detection of the prostate disease types from CT images of abdomen utilizing texture analysis was analyzed in [21]. Texture features were extricated from the images segmented utilizing an evolving transformation called Sequency based Mapped Real Transform (SMRT). This feature sets were obtained by changing and block size and sub-image size. Every feature set was optimized utilizing a Genetic Algorithm (GA). The finest feature set was chosen dependent on accuracy of classification. A fully automated segmentation algorithm for T2-w endorectal prostate MRI was proposed in [22] and assessed its accuracy inside various ROI utilizing a set of complementary error measurements. A neuralfuzzy technique for automatic region segmentation in TRUS images was proposed in [23].

In [26] a non-invasive system for the early recognition of PCa from DW-MRI was analyzed. The prostate was localized and segmented dependent on the new level-set. To maintain sequence, the computed $\mathrm{ADC}$ values were refined and normalized utilizing the image model of Generalized Gauss-Markov Random Field (GGMRF). The multi-layer deeply supervised deconvolution network (DSDN) that executes end-to-end training for automatic segmentation of MRI. More layers deeply supervised were added to supervise the hidden layers performances [27].

\subsection{Classification based Analysis}

The convolutional neural network is one of the feed-forward neural networks, which gives advantages in classification of image undertakings as per practical experience. Among many deep learning algorithms, the $\mathrm{CNN}$ is an algorithm executes better in the classification process. The CNN as an image classification technique that produced some diagnosis classification references as discussed in [28]. A DBN-DS-based multi-classifier prediction model for pathologic stage prostate cancer was used for classification in [29]. The classifiers were made by learning information as indicated by classifier. To assess the DBN-DS-based multi-classifier, the whole dataset was separated into the training and testing sets. In [30], a deep learning system, which depends on the $3 \mathrm{D} \mathrm{CNN}$, to extricate the spatial-temporal features consistently from the images of sequential CEUS was used to detect tumor. For obtaining the CEUS image sequence's temporal patterns, a 3rd dimension was combined in the $\mathrm{CNN}$ model.

A complete automatic CNN-based CAD system for initial diagnoses of $\mathrm{PCa}$ was analyzed in [31]. Prostate delineation depended on the level set which utilized 3 sorts of attributes for enhanced performances. These attributes were shape priors, spatial voxel relationships, and appearance. The integration of the shape priors improved the delineation accuracies as most prostates had analogous structure. These three types of features were combined utilizing a nonnegative matrix factorization (NMF) method. An imaging-based methodology in the prediction of 3 -years biochemical recurrence (BCR) through a novel SVM classifier was analyzed in [32]. AdaBoost-based Ensemble Learning was used in [33] for supporting automatic Gleason grading of prostate adenocarcinoma (PRCD). In [34], an automatic Clinically Significant Prostate Cancer recognition system where every process was optimized together in an end-to-end trainable DNN was analyzed. Three sorts of loss functionalities like classification, overlap, and inconsistency losses were utilized to optimize every parameter of the CNN and TDN. 
Table.2. Analysis of Performance Evaluations

\begin{tabular}{|c|c|c|c|c|c|c|c|}
\hline Author & Year & Performance & Technique & Modality & Dataset & \multicolumn{2}{|c|}{ Results } \\
\hline $\begin{array}{l}\text { Zhiyu Liu et } \\
\text { al. [13] }\end{array}$ & 2019 & $\begin{array}{l}\text { Segmentation } \\
\text { and Classification }\end{array}$ & $\begin{array}{l}\text { Mask R-CNN } \\
\text { and DNN }\end{array}$ & T2W-MRI & $\begin{array}{l}\text { PROSTATEx and } \\
\text { I2CVB }\end{array}$ & AUC & $\begin{array}{l}0.882 \\
0.912\end{array}$ \\
\hline $\begin{array}{l}\text { Haozhe Jia et } \\
\text { al. [16] }\end{array}$ & 2019 & Segmentation & 3D APA-net & T2W-MRI & $\begin{array}{c}\text { Promise12 } \\
\text { ASPS13 } \\
\text { Hybrid } \\
\end{array}$ & DSC & $\begin{array}{l}0.906 \\
0.893 \\
0.901\end{array}$ \\
\hline $\begin{array}{c}\text { Tahereh H et } \\
\text { al. [17] }\end{array}$ & 2019 & Segmentation & FCNN & MRI & Promise12 & DSC & 0.874 \\
\hline $\begin{array}{c}\text { Chuan-Yu C } \\
\text { et al. [18] }\end{array}$ & 2015 & $\begin{array}{l}\text { Feature based } \\
\text { Classification }\end{array}$ & SVM & MRI & Own & $\mathrm{ACC}$ & 94.74 \\
\hline $\begin{array}{c}\text { Shashidhar B } \\
\text { G [19] }\end{array}$ & 2019 & Classification & $\begin{array}{l}\text { Improved } \\
\text { RideNN }\end{array}$ & $\begin{array}{c}\text { Prostate } \\
\text { histopathological } \\
\text { images }\end{array}$ & Own & ACC & 89.66 \\
\hline $\begin{array}{l}\text { Manju B et al. } \\
\text { [20] }\end{array}$ & 2015 & $\begin{array}{l}\text { Feature based } \\
\text { Classification }\end{array}$ & $\begin{array}{c}\text { Genetic } \\
\text { algorithm and } \\
\text { KNN }\end{array}$ & CT & Own & $\mathrm{ACC}$ & 94.30 \\
\hline $\begin{array}{c}\text { Maysam } \\
\text { Shahedi et al. } \\
{[21]}\end{array}$ & 2017 & Segmentation & $\begin{array}{l}\text { Fully automated } \\
\text { segmentation } \\
\text { algorithm }\end{array}$ & T2W-MRI & Own & DSC & 82 \\
\hline $\begin{array}{l}\text { Islam Reda et } \\
\text { al. [22] }\end{array}$ & 2016 & $\begin{array}{c}\text { Segmentation } \\
\text { and Classification }\end{array}$ & $\begin{array}{c}\text { NMF- } \\
\text { Autoencoder }\end{array}$ & DW-MRI & Own & ACC & 97.6 \\
\hline $\begin{array}{c}\text { Ying Liu and } \\
\text { Xiaomei An } \\
{[23]} \\
\end{array}$ & 2017 & Classification & $\mathrm{CNN}$ & DWI-MRI & ImageNet & ACC & 78.15 \\
\hline $\begin{array}{c}\text { Dong Ji et al. } \\
\text { [24] }\end{array}$ & 2018 & Segmentation & DSDN & MRI & $\begin{array}{c}\text { Dataset from Medical } \\
\text { Images Computing } \\
\text { and Computer } \\
\text { Assisted } \\
\text { Interventions }\end{array}$ & DSC & 90.15 \\
\hline $\begin{array}{c}\text { Jae Kwon } \\
\text { Kim et al. [25] }\end{array}$ & 2018 & Classification & DBN-DS & TRUS-Pathology & KPCR & $\begin{array}{l}\text { ACC } \\
\text { AUC }\end{array}$ & $\begin{array}{l}81.27 \\
0.777 \\
\end{array}$ \\
\hline $\begin{array}{l}\text { Yujie Feng et } \\
\text { al. [26] }\end{array}$ & 2018 & $\begin{array}{c}\text { Feature } \\
\text { Extraction and } \\
\text { Classification }\end{array}$ & $3 \mathrm{D}-\mathrm{CNN}$ & CEUS-Ultrasound & CEUS & $\mathrm{ACC}$ & 90.18 \\
\hline $\begin{array}{l}\text { Islam Reda et } \\
\text { al. [27] }\end{array}$ & 2018 & $\begin{array}{c}\text { Feature } \\
\text { Extraction and } \\
\text { Classification }\end{array}$ & $\begin{array}{l}\text { ADCs-based } \\
\text { CNN }\end{array}$ & DWI-MRI & Own & ACC & 97.60 \\
\hline $\begin{array}{c}\text { Yu-Dong } \\
\text { Zhang et al. } \\
{[28]}\end{array}$ & 2016 & Classification & SVM & mp-MRI & Own & ACC & 92.2 \\
\hline $\begin{array}{l}\text { Chao-Hui H } \\
\text { and Emarene } \\
\text { M K [29] }\end{array}$ & 2016 & Classification & $\begin{array}{l}\text { AdaBoost-based } \\
\text { Ensemble } \\
\text { Learning }\end{array}$ & $\begin{array}{c}\text { PRAD } \\
\text { histopathological } \\
\text { image }\end{array}$ & TCGA & $\mathrm{ACC}$ & 97.8 \\
\hline $\begin{array}{c}\text { Zhiwei Wang } \\
\text { et al. [30] }\end{array}$ & 2017 & Classification & TDN and $\mathrm{CNN}$ & mp-MRI & PROSTATEX & AUC & 0.962 \\
\hline $\begin{array}{c}\text { Yanan Shao et } \\
\text { al. [31] }\end{array}$ & 2020 & Classification & GAN & Ultrasound & $\begin{array}{l}\text { CRCEO, JH, PCC, } \\
\text { PMCC, and UVA }\end{array}$ & AUC & 0.934 \\
\hline $\begin{array}{c}\text { R.J.G. van } \\
\text { Sloun et al. } \\
\text { [32] }\end{array}$ & 2019 & $\begin{array}{c}\text { Segmentation } \\
\text { and Classification }\end{array}$ & FCNN & TRUS & Own & $\begin{array}{l}\text { ACC } \\
\text { DSC }\end{array}$ & $\begin{array}{c}98 \\
0.96\end{array}$ \\
\hline $\begin{array}{l}\text { Adeel Ahmed } \\
\text { M et al. [33] }\end{array}$ & 2020 & Classification & $\mathrm{CNN}$ & MRI & $\begin{array}{c}\text { Dataset by Harvard } \\
\text { University }\end{array}$ & AUC & 1.00 \\
\hline
\end{tabular}

\section{DISCUSSION}

Imaging plays a significant role in the prostate cancer detection. Imaging models like mpMRI, mpUS, PET, and MRI-US fusion imaging are utilized in the localization and diagnosis of PCa. For detecting the prostate cancer segmentation and classification are the two fundamental processes to carry out the performance analysis. Prostate segmentation is a difficult process, and the difficulties altogether vary from one modality to another. Micro-calcifications, Low contrast, speckle, and imaging artifacts such as shadow poses are the major difficulties to precisely segment the prostate in medical images. The present $\mathrm{CAD}$ system analyzes images from different imaging modalities like US and MRI for detecting and localizing PCa and also to assess its size and stage. The CAD system is effective and utilized for recognizing $\mathrm{PCa}$ in the central gland (CG), peripheral zone (PZ), and transition zone from MRI T2-weighted. In this study, the detection of PCa based on MRI imaging was considered and reviewed. Further, the CAD processes for $\mathrm{PCa}$ like pre-processing, segmentation, feature extraction and classification is discussed on the plot of MRI based performance. 


\subsection{Imaging Datasets}

The Cancer Imaging Archive (TCIA) has 9 prostate disease data sets accessible from http://www. cancerimagingarchive.net/:

- Prostate-MRI dataset with (26 cases) of prostate MRIs.

- Prostate-Diagnosis project with (92 cases), PCa T1-and T2-w MRIs.

- NaF Prostate with (9 cases) was an assortment of F-18 NaF CT/PET images in patients with $\mathrm{PCa}$.

- The Prostate-3T venture (64 cases) gave Prostate transversal T2-weighted MRIs information to TCIA as a feature of an ISBI challenges competition in 2013.

- The QIN PROSTATE dataset with (22 cases) of the Quantitative Imaging Network (QIN) includes multi-parametric MRIs gathered for the task of detection and staging of prostate cancer.

- The TCGA-PRAD dataset with (14 cases), additionally referenced in the Genomics part of this analysis, and likewise has imaging data (Pathology, CT, PET, and MRI).

- The Prostate Fused-MRI-Pathology dataset with (28 cases) was a fusion of histopathology slides and MRI images.

- The dataset PROSTATEx Challenge with (346 cases) was a prostate review set of MR analysis.

- The dataset QIN-PROSTATE-Repeatability with (15 cases) was a data set with multiparametric PCa MRI implemented in a testretest setting, enabling to assess the MRIbased analysis repeatability in the $\mathrm{PCa}$.

\subsection{Segmentation}

The process of segmentation comprises of representing the MRI's prostate boundaries and specific significance for concentrating the posterior process on organ of interest. The process was to segment the ROIs or prostate from the dataset of $2 \mathrm{D}$ or $3 \mathrm{D}$ prostate data. Different types of datasets are publicly available in three domains: imaging data, clinical data, and genomics data.

This segmentation process could be performed in automatic, self-automatic, or manually. Automated segmentation is a difficult process due to the patient's action or move during image scanning, the inter patients difference of prostate's appearance and shape, inhomogeneities, and noise, disintegration of prostate boundaries as a result of occlusion, and neighboring tissue's comparable intensities.

However the prostate boundary provides the locations of prostate gland volumes while segmenting manually, it needs additional time and was observers based. Accordingly, various segmentation techniques are designed to consider those issues and accurately plot tissues of prostate like deformable and statistical based techniques. The deformable models (DMs) were generally utilized to segment the prostate from DWI. Statistical based techniques were utilized to plot the prostate from MRI methods. The conclusive outcomes, however promising, indicated that the prostate segmentation issue is as yet uncertain.

\subsection{Classification}

The last process of a CAD framework includes training and testing with features extricated from labels and images. Many measurements can be utilized so as to survey the performance of a classifier. The main metric utilized was the accuracy which was calculated as the true identification ratio to the samples count. Though, based upon the technique utilized in the CAD system, this metric could be exceptionally biased through a higher count of true negative instances that would increase the accuracy overrating of the real classifier performance.

The most general statistical computations are specificity and sensitivity which provide a full outline of the classifier's efficiency. Sensitivity was additionally known as true positive value and was equivalent to the samples of true positives ratio beyond true positives included with samples of false negatives. Specificity was likewise known as rate of true negatives and was equivalent to the proportion of the samples of true negatives beyond the true negatives included with samples of false positives. These measurements could be utilized to figure the ROC-(Receiver Operating Characteristic) curves. A statistics obtained from ROC analysis was the AUC- Area Under the Curve that relates to the area under ROC and was an estimation utilized to make comparison among techniques. At last, besides the overlap measures, the Dice's coefficients were normally calculated to assess the lesions localization accuracies.

\section{ANALYSIS}

This segmentation and classification graphical representations are plotted using the results observed from different techniques in the survey. This comparison chart is plotted based on the results obtained from using only MRI images like T2W, DWI, and mp-MRI. Because most of the researches are based on MRI imaging modalities and MRI based research produced best results in terms of detecting prostate cancer efficiently and effectively. In both segmentation and classification the deep learning based techniques performed well with the results. As shown in the figure the $\mathrm{CNN}$ is the top most choice of the 
researchers for segmenting and classifying prostate cancer. The researchers integrate the deep learning techniques with other classifiers to design hybrid techniques for efficient results. The CNN has the advantages of automatically detects the significant features without any manual supervision and it is computationally efficient. Comparing CNN with other techniques like SVM and different machine learning techniques, for example, $\mathrm{CNN}$ is suitable for large datasets where SVM is not and SVM are margin classifier and support different kernels to perform the classification. The deep learning techniques performed better on ultrasound, CT and histopathological images too.

\section{CONCLUSION AND FUTURE WORK}

This survey presents the overview of imaging techniques used for $\mathrm{PCa}$ and the review of segmentation and classification techniques. It was difficult to point out which imaging technique or which classification method is suitable for the PCa detection. Based on the literature review and the analysis of the performance of existing and recent works made on prostate cancer detection, this survey is concluded. Dataset plays an important role in the segmentation and classification process. Different types of datasets are publicly available in three domains: imaging data, clinical data, and genomics data. In the process of detection or classification of $\mathrm{PCa}$, an efficient and accurate algorithm is necessary. Most of the detection techniques are based on CAD- computer aided detection system. In which, preprocessing, segmentation, feature extraction, and classification are the major processes to be carried out for efficient results. Based on this efficient result, this survey is representing the deep learning based classifiers particularly Convolution Neural Network (CNN) was very often used by many researchers for the classification of PCa detection. This type of deep learning classifier was suitable for both segmentation and classification. In future, hybrid techniques based on deep learning and machine learning could be efficient for the task of $\mathrm{PCa}$ detection. A review of $\mathrm{PCa}$, imaging modalities and the techniques used for segmentation and classification of the $\mathrm{PCa}$ was analyzed and surveyed in this paper. This survey will be useful for researchers intended to do a research on detecting and classifying PCa in future.

ETHICS APPROVAL AND CONSENT TO PARTICIPATE

Not applicable.

\section{HUMAN AND ANIMAL RIGHTS}

No animals/humans were used for studies that are basis of this research.

\section{CONSENT FOR PUBLICATION}

Not applicable.

\section{AVAILABILITY OF DATA AND MATERIALS}

The authors confirm that the data supporting the findings of this research are available within the article.

\section{FUNDING}

None.

\section{CONFLICT OF INTEREST}

The authors declare no conflict of interest, financial or otherwise.

\section{ACKNOWLEDGEMENTS}

None.

\section{REFERENCES}

[1] Mary B B. Culp, Isabelle S, Jason A. E, Freddie B, and Ahmedin J. (2019). Recent Global Patterns in Prostate Cancer Incidence and Mortality Rates. Eur Urol. Elsevier. pp.1-15.

[2] Jean-L D. (2019). Diagnosis of prostate cancer. Asian $J$ Urol. Science Direct. Vol.6, pp.129-136.

[3] Raman P S, Savita G, Rajendra A. (2017). Segmentation of prostate contours for automated diagnosis using ultrasound images: A survey. J Comput Sci. Elsevier. pp.1-31.

[4] Ahmed S et al. (2018). Computer-Aided Diagnosis of Prostate Cancer on Diffusion Weighted Imaging: A Technical Review. IEEE International Conference on Imaging Systems and Techniques (IST). pp.1-6.

[5] Hüseyin C D and John W D. (2018). Multiparametric magnetic resonance imaging: Overview of the technique, clinical applications in prostate biopsy and future directions. Turk J Urol. Vol.44, No.2, pp.93-102.

[6] Tristan B. (2015). What is multiparametric-MRI of the prostate and why do we need it? Imag Med. Vol.7, No.2, pp.13-17.

[7] Bejoy A and Madhu S N. (2019) On Computer-Aided Diagnosis of Prostate Cancer from MRI using Machine Intelligence Techniques. IEEE International Conference on Electrical, Computer and Communication Technologies. pp.1-8.

[8] Latrach A, Trigui R, Chenini H, Sellemi L, and Ben H A. (2018). Comparison Study for Computer Assisted Detection and Diagnosis 'CAD' systems Dedicated to Prostate Cancer Detection Using MRImp Modalities. International Conference on Advanced Technologies for Signal and Image Processing. IEEE. 2018, pp.1-6.

[9] Saradwata S and Sudipta D. (2016). A Review of Imaging Methods for Prostate Cancer Detection. Biomed Eng Comput Biol. Vol.7, No.S1, pp.1-15.

[10] Andreas $\mathrm{M}$ and Thomas F. Clinical value of multiparametric ultrasound and MRI/US fusion-guided biopsy for prostate cancer detection and visualization. Canon Medical Systems. 
[11] Andrea B et al. (2018). A review on the role of water Diffusion modeling in Magnetic Resonance Imaging of Prostate cancer. IEEE Workshop on Complexity in Engineering (COMPENG). pp. 1-5.

[12] Alexander S L and Arvid L. (2019). An overview of deep learning in medical imaging focusing on MRI. $Z$ Med Phys. Elsevier. Vol.29, pp.102-127.

[13] Zhiyu L et al. (2019). A Two-Stage Approach for Automated Prostate Lesion Detection and Classification with Mask R-CNN and Weakly Supervised Deep Neural Network. Artif Intell Radiat Ther. Springer. pp.43-51.

[14] Guillaume L, Robert M, Jordi F, Joan C. V, Paul M. W, and Fabrice M. (2015). Computer-Aided Detection and diagnosis for prostate cancer based on mono and multi-parametric MRI: A review. Comput Biol Med. Elsevier. Vol.60, pp-1-54.

[15] Shijun W, Karen B, Baris T, Peter C, and RonaldM S. (2014). Computer Aided-Diagnosis of Prostate Cancer on Multiparametric MRI: A Technical Review of Current Research. BioMed Research International. Hindawi. Vol.2014, pp.1-11.

[16] Haozhe J, Yong X, Yang S, Donghao Z, Heng H, Yanning Z, and Weidong C. (2020). 3D APA-Net: 3D Adversarial Pyramid Anisotropic Convolutional Network for Prostate Segmentation in MR Images. IEEE T Med Imaging. Vol.39, No.2, pp.447-457.

[17] Tahereh H, Len H, and Kevin H S. (2016). Convolutional Neural Networks for Prostate Magnetic Resonance Image Segmentation. IEEE Access. Vol.4, pp.1-12.

[18] Chuan-Yu C, Hui-Ya H, and Yuh-Shyan T. (2015). Prostate Cancer Detection in Dynamic MRIs. IEEE International Conference on Digital Signal Processing (DSP). pp.1279-1282.

[19] Shashidhar B. G, Kshama V. K, and Veena V. D. Prostate Cancer Detection using Histopathology Images and Classification using Improved RideNN. Biomed Eng-App Bas C. Vol.31, No.6, pp.1-14.

[20] Manju B, K.Meenakshy, and R. Gopikakumari. (2015). Prostate Disease Diagnosis from CT images using GA optimized SMRT based Texture Features. International Conference on Information and Communication Technologies. Procedia Computer Science. Vol.46, pp.1692-1699.

[21] Maysam Shahedi et al. (2017). Accuracy Validation of an Automated Method for Prostate Segmentation in Magnetic Resonance Imaging. J digit imaging. Vol.30, pp.782-795.

[22] Islam Reda et al. (2016). A New NMF-Autoencoder based CAD System for Early Diagnosis of Prostate Cancer. IEEE 13th International Symposium on Biomedical Imaging (ISBI). pp.1237-1240.

[23] Ying L and Xiaomei A. (2017). A Classification Model for the Prostate Cancer Based on Deep Learning. International Congress on Image and Signal Processing. BioMed Eng Inform. IEEE. pp.1-6.

[24] Dong J, Jun Y, Toru K, Liangfeng X, and Shu Z. (2018). Automatic Prostate Segmentation on MR Images with Deeply Supervised Network. International Conference on Control. Decision and Information Technologies. IEEE. pp.309-314.
[25] Jae K K et al. (2018). A Deep Belief Network and Dempster-Shafer-Based Multiclassifier for the Pathology Stage of Prostate Cancer. $J$ Healthc Eng. Hindawi. Vol.2018, pp.1-8.

[26] Yujie F, Fan Y, Xichuan Z, Yanli G, Fang T, Fengbo R, Jishun G, and Shuiwang J. (2019). A Deep Learning Approach for Targeted Contrast-Enhanced Ultrasound Based Prostate Cancer Detection. IEEE/ACM T Comput Biol Bioinform. Vol. 16, No. 6, pp. 1794-1801.

[27] Islam R et al. (2018). A Novel ADCs-Based CNN Classification System for Precise Diagnosis of Prostate Cancer. International Conference on Pattern Recognition (ICPR). IEEE. pp.3923-3928.

[28] Yu-Dong $\mathrm{Z}$ et al. (2016). An imaging-based approach predicts clinical outcomes in prostate cancer through a novel support vector machine classification. Oncotarget. Vol.7, No.47, pp.78140-78151.

[29] Chao-Hui H and Emarene M K. (2016). Automated Classification for Pathological Prostate Images using AdaBoost-based Ensemble Learning. IEEE Symposium Series on Computational Intelligence (SSCI). pp. 1-4

[30] Zhiwei W, Chaoyue L, Danpeng C, Liang W, Xin Y, and K.T. Tim Cheng. (2018). Automated Detection of Clinically Significant Prostate Cancer in mp-MRI Images based on an End-to-End Deep Neural Network. IEEE T Med Imaging. vol. 37, no. 5, pp. 1127-1139.

[31] Yanan S, Jane W, Brian W, and Septimiu E S. (2020) Improving Prostate Cancer (PCa) Classification Performance by Using Three-Player Minimax Game to Reduce Data Source Heterogeneity. IEEE T Med Imaging. pp.1-11.

[32] R.J.G. van Sloun et al. (2018). Zonal segmentation in transrectal ultrasound images of the prostate through deep learning. IEEE International Ultrasonics Symposium (IUS). pp.1-4.

[33] Adeel A A et al. (2020). Detecting prostate cancer using deep learning convolution neural network with transfer learning approach. Cogn Neurodynamics. pp.1-11. 